\title{
Bovine Papillomavirus Type 2 Infection and Microscopic Patterns of Urothelial Tumors of the Urinary Bladder in Water Buffaloes
}

\author{
Paola Maiolino, ${ }^{1}$ Ayhan Özkul, ${ }^{2}$ Aylin Sepici-Dincel, ${ }^{3}$ Franco Roperto, ${ }^{4}$ Gözde Yücel, ${ }^{2}$ \\ Valeria Russo, ${ }^{1}$ Chiara Urraro, ${ }^{1}$ Roberta Lucà, ${ }^{1}$ Marita Georgia Riccardi, ${ }^{1}$ \\ Manuela Martano, ${ }^{1}$ Giuseppe Borzacchiello, ${ }^{1}$ Iolanda Esposito, ${ }^{1}$ and Sante Roperto ${ }^{1}$ \\ ${ }^{1}$ Department of Veterinary Medicine and Animal Productions, Naples University Federico II, Via Delpino 1, 80137 Naples, Italy \\ ${ }^{2}$ Department of Pathology, Faculty of Veterinary Medicine, Ankara University, 06110 Ankara, Turkey \\ ${ }^{3}$ Faculty of Medicine, Gazi University, 06100 Ankara, Turkey \\ ${ }^{4}$ Department of Biology, Naples University Federico II, 80134 Naples, Italy
}

Correspondence should be addressed to Valeria Russo; valeria.russo@unina.it

Received 22 February 2013; Accepted 22 April 2013

Academic Editor: Rita de Cassia Stocco

Copyright (C) 2013 Paola Maiolino et al. This is an open access article distributed under the Creative Commons Attribution License, which permits unrestricted use, distribution, and reproduction in any medium, provided the original work is properly cited.

\begin{abstract}
Microscopic patterns of thirty-four urothelial tumors of the urinary bladder of water buffaloes from the Marmara and Black Sea Regions of Turkey are here described. All the animals grazed on lands rich in bracken fern. Histological diagnosis was assessed using morphological parameters recently suggested for the urinary bladder tumors of cattle. Papillary carcinoma was the most common neoplastic lesion (22/34) observed in this study, and low-grade carcinoma was more common (seventeen cases) than high-grade carcinoma (five cases). Papilloma, papillary urothelial neoplasm of low malignant potential (PUNLMP), and invasive carcinomas were less frequently seen. Carcinoma in situ (CIS) was often detected associated with some papillary and invasive carcinomas. De novo (primary) CIS was rare representing 3\% of tumors of this series. A peculiar feature of the most urothelial tumors was the presence in the tumor stroma of immune cells anatomically organized in tertiary lymphoid organs (TLOs). Bovine papillomavirus type- 2 (PV-2) E5 oncoprotein was detected by molecular and immunohistochemistry procedures. Early protein, E2, and late protein, L1, were also detected by immunohistochemical studies. Morphological and molecular findings show that BPV-2 infection contributes to the development of urothelial bladder carcinogenesis also in water buffaloes.
\end{abstract}

\section{Introduction}

Spontaneous tumors of the urinary bladder are very rare in cattle accounting for $0.01 \%$ of all bovine malignancies [1]. Conversely, they are common in adult cattle grazing on lands rich in bracken fern [2-4]. This plant contains toxic substances impairing the immune system and carcinogen principles such as ptaquiloside, the prolonged ingestion of which appears to be involved in bladder carcinogenesis. It has been suggested that ptaquiloside causes an increased cell proliferation in bladder urothelium resulting in urothelial dysplasia $[5,6]$; furthermore, it is believed that ptaquiloside can act synergistically with bovine papillomavirus type 2 (BPV-2) thus causing bladder tumors in cattle [7].
Papillomavirus infection plays a central role in bladder carcinogenesis of large ruminants [7-11]. In particular, BPV2 appears to be involved in many urothelial tumors in cattle and water buffaloes $[4,7,12]$. It has been shown that BPV-2 causes in vivo bladder carcinogenesis through the activation of PDGF $\beta$ receptor $[12,13]$ and/or of Calpain 3 which is responsible for urothelial cell proliferation via E2F3 protein [14].

Tumors of the urinary bladder of buffaloes have sporadically been described [3]. However, BPV-2 infection has just been reported in urothelial tumors as well as in some nonneoplastic lesions of the urinary bladder of buffaloes [12].

The aim of the present paper is to report the microscopic patterns of thirty-four urothelial tumors of the urinary bladder of water buffaloes, twenty-seven of which were associated 
with papillomavirus infection. All the animals were from the Marmara and Black Sea Regions of Turkey and grazed on pastures contaminated with bracken fern.

\section{Materials and Methods}

Thirty-four tumor samples of the urinary bladder were collected at public slaughterhouses of Marmara and Black Sea Region (Turkey) (Bafra, Coskun, Bartin) from 3- to 5-yearold castrated male water buffaloes daily grazing on ferninfested lands. Each sample was divided into two halves. One part was fixed in $10 \%$ neutral buffered formalin and was processed for paraffin embedding for morphological assessment; the other half was immediately frozen in liquid nitrogen, stored at $-80^{\circ} \mathrm{C}$ until further processed for molecular procedures.

2.1. Histopathology and Immunohistochemistry. Histologic diagnosis of urinary bladder tumors was assessed on $4-\mu \mathrm{m}$ thick hematoxylin-eosin- (HE-) stained sections using morphological criteria suggested in a recent report on the new histological classification of urothelial tumors of the urinary bladder of cattle [4]. Immunohistochemistry was performed to detect both early (E) and late (L) papillomavirus proteins. Briefly, the sections were deparaffinized, and then endogenous peroxidase activity was blocked by incubation in $0.3 \%$ $\mathrm{H}_{2} \mathrm{O}_{2}$ in methanol for $20 \mathrm{~min}$. Antigen retrieval was performed by pretreating with microwave heating (twice for $5 \mathrm{~min}$ each at $750 \mathrm{~W}$ ) in citrate buffer $\mathrm{pH}$ 6.0. The slides were washed with phosphate buffered saline (PBS), pH 7.4, 0.01 M, then incubated for $1 \mathrm{~h}$ at room temperature with donkey serum (Sigma-Aldrich, Milan, Italy) diluted at 1 in 10 in PBS. A polyclonal sheep anti-BPV-2 E5 primary antibody (a kind gift from Dr. M. S. Campo, professor Emeritus of Glasgow University, UK) diluted at 1 in 40000 in PBS was applied for $1 \mathrm{~h}$ at room temperature in a humid chamber. The sections were rinsed with PBS before application of the donkey antisheep biotinylated secondary antibody (Santa Cruz Biotechnology Inc., CA, USA), diluted at 1 in 100 in PBS for 45 min at room temperature. For E2 and L1 detection, the slides were washed three times with PBS, $\mathrm{pH} 7.4,0.01 \mathrm{M}$, then incubated for $1 \mathrm{~h}$ at room temperature with protein block serum free (DakoCytomation, Denmark). A polyclonal rabbit anti-BPV2 E2 primary antibody (a kind gift from Dr. E. Androphy) diluted at 1 in 50 in PBS and a monoclonal mouse anti-HPV16 L1 (late protein) primary antibody diluted at 1 in 200/300 in PBS were applied overnight at $4^{\circ} \mathrm{C}$ in a humid chamber. The sections were rinsed three times for $5 \mathrm{~min}$ with PBS, incubated for $40 \mathrm{~min}$ at room temperature with appropriate biotinylated secondary antibody (labelled streptavidin-biotin (LSAB) Kit; DakoCytomation, Denmark). Finally, all the sections were washed with PBS and then incubated with streptavidin-conjugated to horseradish peroxidase (LSAB Kit; DakoCytomation, Denmark).

Color development was obtained by treatment with diaminobenzidine (DakoCytomation, Denmark) for 5-20 min. Sections were counterstained with Mayer's hematoxylin. Negative control sections were incubated with PBS instead of primary antibody.
2.2. PCR Reaction. The DNA was extracted from the urinary bladders of water buffaloes using the DNeasy Tissue Kit (Qiagen TM, Germany) according to the manufacturer's instructions.

For the detection of BPV-2 E5 DNA, specific primers for the E5 ORF (forward primer, $5^{\prime}$-CACTGCCATTTGTTTTTTTC- ${ }^{\prime}$; reverse primer, $5^{\prime}$-GGAGCACTCAAAATGATCCC- $3^{\prime}$ ) were used. Furthermore, specific primers for the $\mathrm{L} 1$ region of the BPV-1 (forward primer, $5^{\prime}$-GGCTGAGGACGCTGCTGGTA-3'; reverse primer, 5' ${ }^{\prime}$-TCTCCGAGCCCCCTCTGGTC- $3^{\prime}$ ) were also employed. Aliquots 50$100 \mathrm{ng}$ of purified DNA were amplified in $25 \mu \mathrm{L}$ of reaction mixture containing $2 \mathrm{mM} \mathrm{MgCl}_{2}, 200 \mathrm{mM}$ each dNTP, $480 \mathrm{nM}$ of each primer, and $2.5 \mathrm{U}$ of AmpliTaq Gold DNA Polymerase (Applied Biosystems, Monza, Italy). The reaction was carried out in a thermocycler (Veriti, Applied Biosystems) with an initial denaturation step of 3 min. Then, 35 cycles of amplification were carried out with a denaturation step at $95^{\circ} \mathrm{C}$ for $45 \mathrm{sec}$, an annealing step at $50^{\circ} \mathrm{C}, 45 \mathrm{sec}$, for BPV-2 E5, or at $65^{\circ} \mathrm{C}, 40 \mathrm{sec}$, for BPV-1 L1 and an extension step at $72^{\circ} \mathrm{C}$ for $1 \mathrm{~min}$. A final extension step at $72^{\circ} \mathrm{C}$ for $7 \mathrm{~min}$ was performed. Detection of the amplified products was carried out by electrophoresis on ethidium bromide-stained agarose gel. In each experiment, a blank sample consisting of reaction mixture without DNA and a positive control composed of BPV-2 clone DNA (a kind gift from Dr. A. Venuti) or of BPV-1 positive sample were included. One $\mu \mathrm{L}$ of the amplified products was subjected to a second run of PCR under the same experimental conditions. Amplified products from the last PCR were electrophoresed in a 2,5\% agarose gel and visualized by ethidium bromide stain.

2.3. RNA Extraction. Total RNA was extracted from urinary bladders of water buffaloes using the RNeasy Mini Kit (Qiagen TM, Germany), according to the manufacturer's instructions. The RNA quality was determined by agarose gel electrophoresis and ultraviolet spectrophotometer analysis.

2.4. cDNA-E5 Analysis. The reverse transcription reaction contained $4 \mu \mathrm{L} 5 \mathrm{x}$ script reaction mix, $1 \mu \mathrm{L}$ iScript reverse transcriptase, and $500 \mathrm{ng}$ total RNA as the template. The volume was adjusted to $10 \mu \mathrm{L}$ with RNase free water (BioRad Laboratories, Milan, Italy). The reaction was incubated at $25^{\circ} \mathrm{C}$ for $5 \mathrm{~min}, 42^{\circ} \mathrm{C}$ for $30 \mathrm{~min}, 85^{\circ} \mathrm{C}$ for $5 \mathrm{~min}$, and then kept at $4^{\circ} \mathrm{C}$ for $5 \mathrm{~min}$. The synthesized cDNA was analyzed by PCR with specific primers for the E5 ORF (forward primer, $5^{\prime}$-CACTGCCATTTGTTTTTTTC- $3^{\prime}$; reverse primer, $5^{\prime}$ GGAGCACTCAAAATGATCCC- $3^{\prime}$ ). The total reaction volume was $25 \mu \mathrm{L}$ containing $2.5 \mu \mathrm{L} 10 \mathrm{x}$ Gold Buffer, $2 \mathrm{mM}$ $\mathrm{MgCl}_{2}, 200 \mathrm{mM}$ each dNTP, $480 \mathrm{nM}$ of each primer, $2.5 \mathrm{U}$ of AmpliTaq Gold DNA Polymerase (Applied Biosystems, Monza, Italy), and $100 \mathrm{ng}$ reverse transcription products of cDNA as the template. The reaction procedure was as follows: predenaturation at $95^{\circ} \mathrm{C}$ for $3 \mathrm{~min}, 35$ cycles (denaturation at $95^{\circ} \mathrm{C}$ for $45 \mathrm{sec}$, annealing at $50^{\circ} \mathrm{C}$ for $45 \mathrm{sec}$, and extension at $72^{\circ} \mathrm{C}$ for $1 \mathrm{~min}$ ), and the final extension at $72^{\circ} \mathrm{C}$ for $7 \mathrm{~min}$. Detection of the amplified products was carried out by electrophoresis on ethidium bromide-stained agarose 


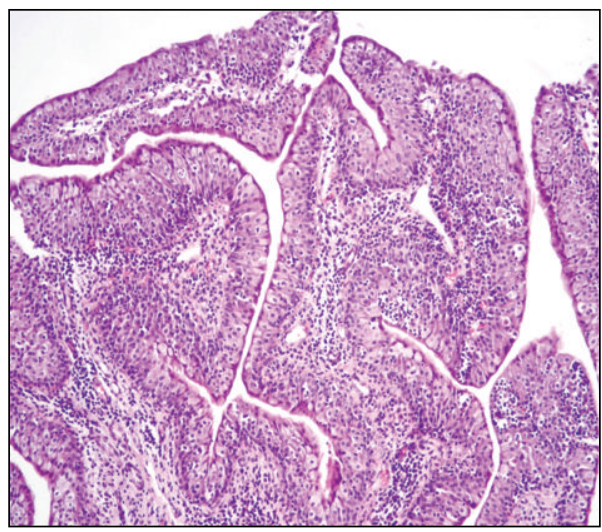

(a)

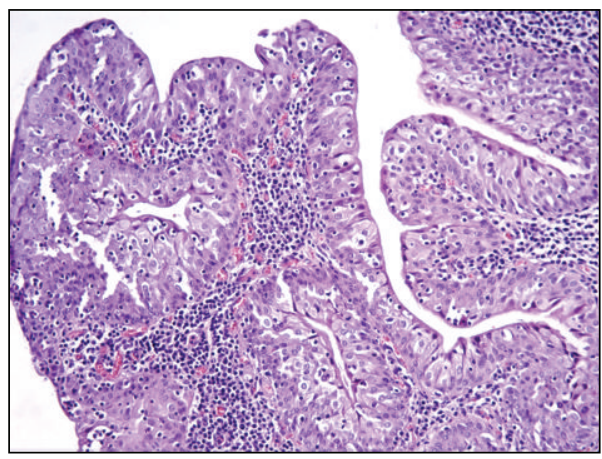

(c)

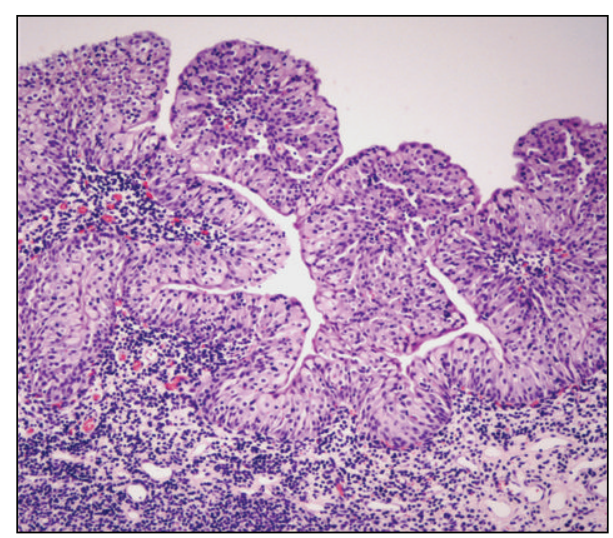

(b)

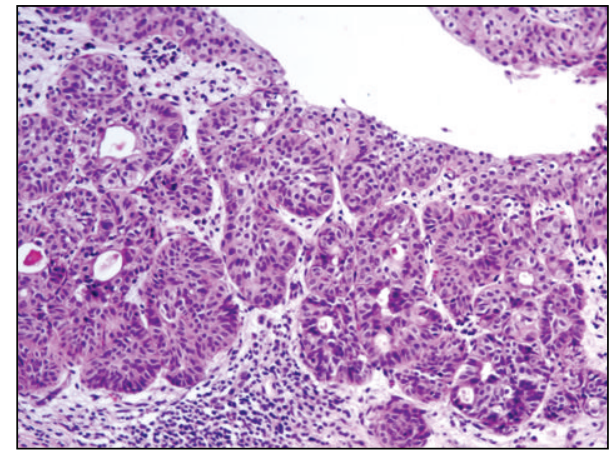

(d)

Figure 1: Morphological patterns of the bubaline urothelial tumors. Papilloma (a), H\&E, Objective 10x; papillary urothelial neoplasm of low malignant potential (PUNLMP). Notice the diffuse reactive atypia, that is, the presence inflammatory cells into the urothelium (b) H\&E, Objective 10x; papillary carcinoma (c) H\&E, Objective 20x; invasive carcinoma (d) H\&E, Objective 20x.

gel. In each experiment, a blank sample consisting of reaction mixture without DNA and a positive sample consisting of cloned BPV-2 (a kind gift from Dr. A. Venuti) were included.

\section{Results}

Histological examinations of the urinary bladder tumors detected microscopic patterns consistent with the diagnosis of urothelial papilloma in three cases, two of them showed an exophytic pattern and only one showed an inverted growth. Furthermore, three cases were diagnosed as papillary urothelial neoplasm of low malignant potential (PUNLMP); a diagnosis of papillary carcinoma was performed in twentytwo cases; five cases of invasive carcinomas were also found (Figure 1); de novo (primary) carcinoma in situ (CIS) was seen in only one case (Figure 2). Table 1 summarizes the results. Tumor-like lesions such as reactive atypia as well as flat and nodular hyperplasia were also seen.

Papillary carcinoma was the most common neoplastic lesion observed in this study, and low-grade carcinomas were more common (seventeen cases) than high grade carcinomas (five cases). CIS was seen to occur in three buffaloes with papillary carcinoma and in two with invasive bladder neoplasia. They were seen more frequently than the primary (de novo) CIS; the latter was seen in only one case thus representing

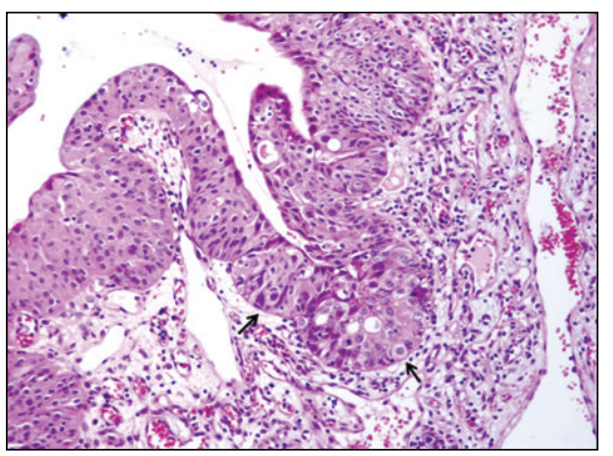

Figure 2: De novo (primary) carcinoma in situ (CIS) H\&E, Objective 20x.

about $3 \%$ of urothelial neoplasms. In all the examined cases, there was a strong inflammation of the tumor stroma; quite frequently, immune cells were anatomically organized in tertiary lymphoid organs (TLOs), in which clear germinal center and peripheral small dark and densely packed mantle lymphocytes without any fibrous encapsulation were seen (Figure 3).

PCR analysis demonstrated the presence of E5 DNA in the samples from twenty-seven buffaloes suffering from uro- 
TABLE 1: Histological types of thirty-four urothelial tumors of the urinary bladder in water buffaloes.

\begin{tabular}{lc}
\hline Microscopic patterns of tumors & Case no. \\
\hline Papilloma & 3 \\
$\begin{array}{l}\text { Papillary urothelial neoplasm of low malignant } \\
\text { potential (PUNLMP) }\end{array}$ & 3 \\
Low-grade papillary urothelial carcinoma & 17 \\
High-grade papillary urothelial carcinoma & 5 \\
Low-grade invasive urothelial carcinoma & 3 \\
High-grade invasive urothelial carcinoma & 2 \\
De novo carcinoma in situ (CIS) & 1 \\
\hline
\end{tabular}

thelial bladder tumors in which a fragment of the expected size (154 bp) was amplified (Figure 4). Furthermore, in these samples, reverse transcriptase PCR analysis demonstrated the presence of E5 mRNA (Figure 5). No BPV-1 DNA was detected. Immunohistochemical studies confirmed the presence of E5 protein in the cytoplasm of many neoplastic cells; it was not detected in the cytoplasm of normal cells (Figure 6). Furthermore, a marked immunoreactivity for L1 protein expression was evident both in the cytoplasm and nuclei of the urothelial cells of neoplastic nests; it was not detectable in normal urothelial cells (Figure 7). Since it is known that E2 protein is essential for the viral life cycle and plays a part in productive infection, immunohistochemical studies on the expression of this protein were also carried out. E2 protein expression was detected both in the cytoplasm and nuclei of urothelial cells from neoplastic urinary bladder. Its expression was not manifested in normal urothelial cells (Figure 8).

\section{Discussion}

The present study reports the microscopic patterns of thirtyfour spontaneous urothelial tumors of the urinary bladder in water buffaloes grazed on pasturelands rich in fern. A papillomavirus infection has been shown in twenty-seven tumors $(\sim 79 \%)$. Both benign and malignant lesions here described fell into the following categories: flat urothelial lesions, papillary urothelial lesions, and invasive urothelial tumors just reported in humans [15] and cattle [4]. No metastases were found in malignant tumors. Our findings corroborate that morphological criteria suggested for histological classification of urothelial tumors in cattle [4] may be used also in water buffaloes. Recently, we have shown that BPV-2 plays a central role also in bubaline bladder carcinogenesis. Like the case in bladder carcinogenesis of cattle, BPV-2 E5 oncoprotein binds to the activated form of platelet derived growth factor $\beta$ receptor (PDGF $\beta \mathrm{R}$ ) [12]. We document here a papillomavirus infection by western blot analysis, cDNA, and immunohistochemical investigations about the presence of BPV-2 E5 oncoprotein. The expression of BPV-2 L1 protein indicates that a complete life cycle of BPV-2 occurs also in bubaline neoplastic urothelial cells. These findings corroborate our previous results about the crucial role of BPV-2

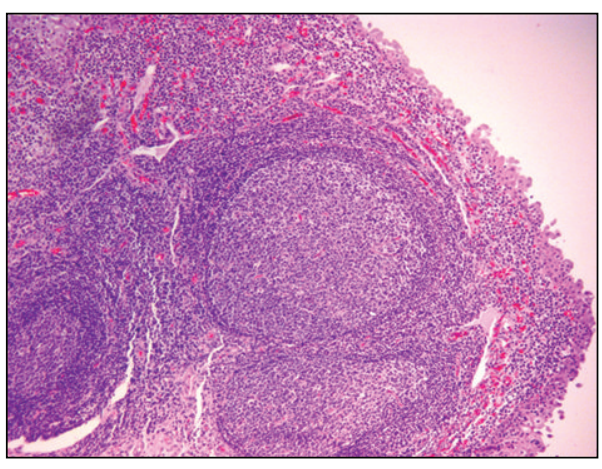

Figure 3: Lymphoid neogenesis. Tertiary lymphoid organs with an evident, clear germinal center and peripheral lymphocytes are evident in tumor stroma. H\&E, Objective 10x.

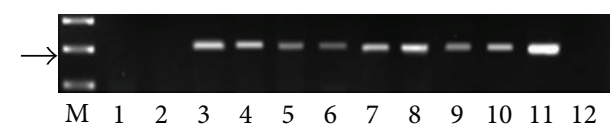

Figure 4: Detection of BPV-2 E5 DNA. M: 50 bp molecular marker (HyperLadder II Bioline); 1-2: urinary bladder samples from healthy buffaloes without BPV-2 E5 DNA; 3-10: some representative bladder tumors samples showing BPV-2 E5 DNA; 11: positive control (cloned BPV-2 DNA); 12: negative control (no DNA added). The arrow indicates the position of the $154 \mathrm{bp}$ BPV-2 E5 PCR product.

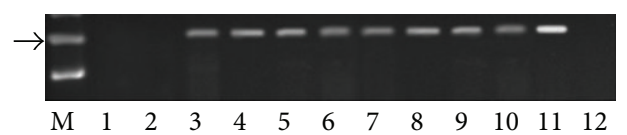

FIGURE 5: Assessment of BPV-2 E5 cDNA by reverse transcriptase polymerase chain reaction (RT-PCR). M: $50 \mathrm{bp}$ molecular marker (HyperLadder II Bioline); 1-2: healthy urinary bladder samples; 3-10: bladder tumors samples; 11: positive control (cloned BPV-2 DNA); 12: negative control (no DNA added). The arrow indicates the position of the $154 \mathrm{bp}$ BPV-2 E5 PCR product.

infection in urothelial cell transformation [12]. Toxic elements of the fern appear to play a synergistic role with BPV-2 infection also in bladder carcinogenesis of water buffaloes. As a matter of fact, when the buffalo breeding based on closed breeding techniques does not allow buffaloes to graze on pastures contaminated with bracken fern, the bladder disease is basically unknown although clinical cases of BPV infection can be seen, that is, papillomatosis of the skin. The microscopical findings of this study show that urothelial tumors of buffaloes share morphological characteristics with the human counterparts just like urothelial tumors of cattle [4, $15,16]$. A peculiar feature of most bubaline urothelial tumors was the presence of diffuse lymphocytes, plasma cells, and macrophages which can evolve into aggregates in the tumor stroma. These findings were also detected in the urothelial cancers of cattle [4], but in buffaloes the immune cells were often organized anatomically in de novo follicles leading to the formation of the so-called tertiary lymphoid organs (TLOs). Chronic follicular cystitis is frequently found in water buffaloes [17]. The pathophysiological significance of 


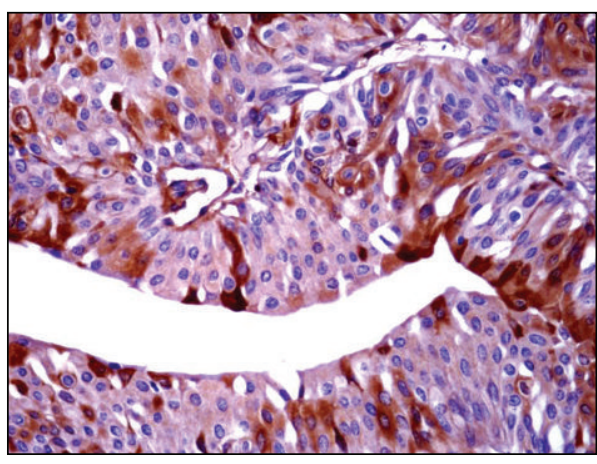

(a)

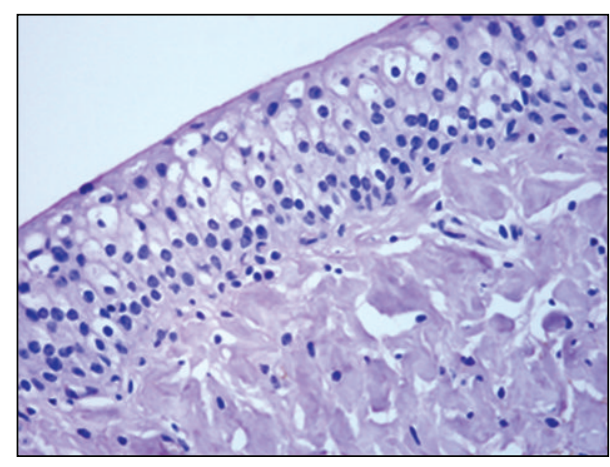

(b)

Figure 6: E5 immunohistochemistry. Neoplastic cells show a strong cytoplasmatic E5 immunoreactivity (a), Objective 40x. No E5 immunoreactivity is evident in normal urothelial cells (b), Objective 20x.

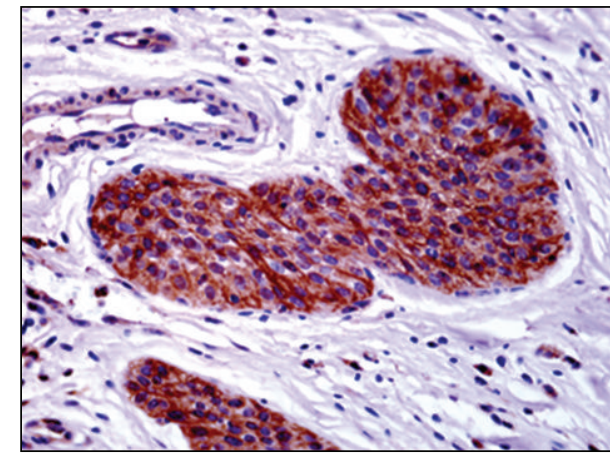

(a)

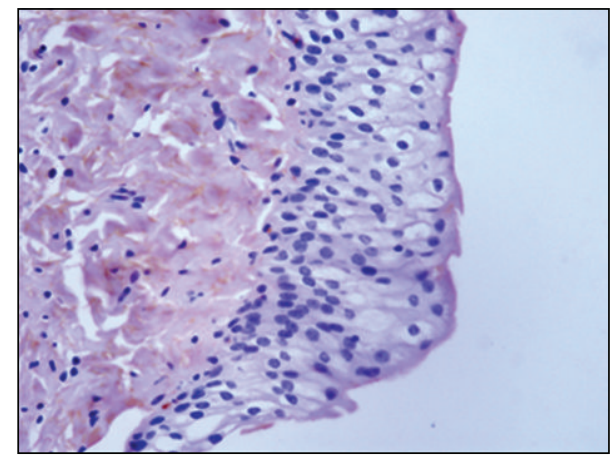

(b)

FIGURE 7: L1 immunohistochemistry. Presence of L1 protein in urothelial neoplastic cells (a) Objective 40x. No L1 protein immunoreactivity was detected in normal urothelial cells (b), Objective 40x.

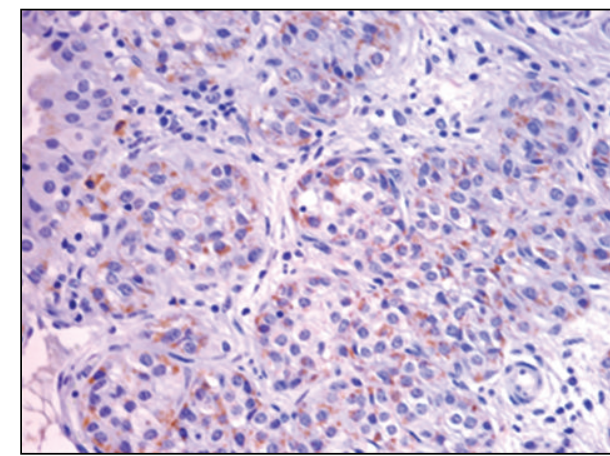

(a)

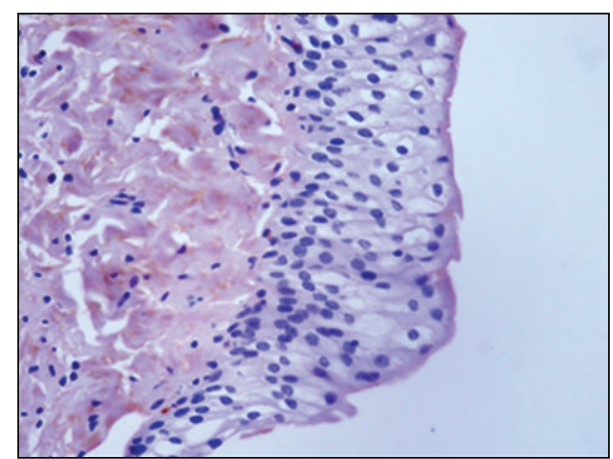

(b)

FIGURE 8: E2 immunohistochemistry. Diffuse immunoreactivity of E2 protein in urothelial neoplastic cells (a), Objective 40x. No E2 protein immunoreactivity was detected in normal urothelial cells (b), Objective 40x.

the lymphoid neogenesis is still unclear. TLO formation is now recognized as a common feature of many chronic inflammatory diseases and might have a role in maintaining immune responses against persistent antigens. It has been suggested that the stimulus that triggers lymphoid neogenesis in infected tissues is the causative agent itself [18]. It is reasonable to suggest that, in our cases, L1 protein, expressed only in productive BPV-2 infection and known to play a central role both in infection and immunogenicity [19], stimulates adaptive immune responses thus leading to chronic inflammation of tumor stroma in which TLOs can develop. Furthermore, BPV E5 is known to impair the major histocompatibility class I complex (MHC I) thus allowing the persistence of the virus infection. This impairment and immunosuppressant of bracken fern may help maintain antigen stimuli responsible for a heavy cell infiltration which can 
contribute to TLO formation $[7,20]$. It has been suggested that chronic follicular cystitis in buffaloes is characterized by a very strong presence of lymphoid cells [17]. It is worth remembering that the most organized structures are generally found in highly infiltrated tissues [18]. Inflammatory cells of microenvironment and their secreted factors appear to be coresponsible for the fate of a tumor [21] being able to exert a strong anticancer immunosurveillance. Further studies are needed to gather insights into the relationship between tumor cells and their inflammatory microenvironment being a manner of debate [22]. In addition, we have to better understand the role of lymphoid neogenesis in infectious diseases as it has been suggested that, although so far it has been documented in only a few chronic diseases, lymphoid neogenesis might have a protective function by inducing an immune response against the infectious agent.

\section{Conflict of Interests}

The authors declare that they have no conflict of interests.

\section{Acknowledgments}

The authors thank Dr. M. S. Campo, Professor Emeritus of Glasgow University, for providing them with the E5 primary antibody; Dr. E. Androphy, University of Massachusetts Medical School, for providing them with the E2 primary antibody; Dr. A. Venuti, Laboratory of Virology, National Cancer Institute "Regina Elena," for giving them the BPV-2 DNA plasmid; Dr. G. Salvatore, Regione Basilicata for his technical help. This work was sponsored by grants from Ministero Italiano dell'Università e della Ricerca Scientifica (MIUR), Ministero Italiano delle Politiche Agricole, Alimentari e Forestali (MIPAAF), and Assessorato alla Sanità della Regione Basilicata.

\section{References}

[1] D. J. Meuten, "Tumors of the urinary bladder and urethra," in Tumors in Domestic Animals, D. J. Meuten, Ed., pp. 524-546, Iowa State Press, Ames, Iowa, USA, 2002.

[2] A. M. Pamukcu, J. M. Price, and G. T. Bryan, "Naturally occurring and bracken fern induced bovine urinary bladder tumors. Clinical and morphological characteristics," Veterinary Pathology, vol. 13, no. 2, pp. 110-122, 1976.

[3] I. A. Özkul and Y. Aydin, "Tumours of the urinary bladder in cattle and water buffalo in the black sea region of Turkey," British Veterinary Journal, vol. 152, no. 4, pp. 473-475, 1996.

[4] S. Roperto, G. Borzacchiello, R. Brun et al., "A review of bovine urothelial tumours and tumour-like lesions of the urinary bladder," Journal of Comparative Pathology, vol. 142, no. 2-3, pp. 95-108, 2010.

[5] R. M. Gil da Costa, M. M. Bastos, P. A. Oliveira, and C. Lopes, "Bracken-associated human and animal health hazards: chemical, biological and pathological evidence," Journal of Hazardous Materials, vol. 203-204, pp. 1-12, 2012.

[6] R. M. Gil da Costa, P. A. Oliveira, M. M. Bastos, C. C. Lopes, and C. Lopes, "Ptaquiloside-induced early-stage urothelial lesions show increased cell proliferation and intact $\beta$-catenin and ecadherin expression," Environmental Toxicology, 2012.
[7] M. S. Campo, W. F. H. Jarrett, R. Barron, B. W. O’Neil, and K. T. Smith, "Association of bovine papillomavirus type 2 and bracken fern with bladder cancer in cattle," Cancer Research, vol. 52, no. 24, pp. 6898-6904, 1992.

[8] G. Borzacchiello, G. Lovane, M. L. Marcante et al., "Presence of bovine papillomavirus type 2 DNA and expression of the viral oncoprotein E5 in naturally occurring urinary bladder tumours in cows," Journal of General Virology, vol. 84, no. 11, pp. 29212926, 2003.

[9] S. R. Wosiacki, M. P. Claus, A. F. Alfieri, and A. A. Alfieri, "Bovine papillomavirus type 2 detection in the urinary bladder of cattle with chronic enzootic haematuria," Memorias do Instituto Oswaldo Cruz, vol. 101, no. 6, pp. 635-638, 2006.

[10] S. Roperto, R. Brun, F. Paolini et al., "Detection of bovine papillomavirus type 2 in the peripheral blood of cattle with urinary bladder tumours: possible biological role," Journal of General Virology, vol. 89, no. 12, pp. 3027-3033, 2008.

[11] S. Roperto, S. Comazzi, E. Ciusani et al., "PBMCS are additional sites of productive infection of bovine papillomavirus type 2," Journal of General Virology, vol. 92, no. 8, pp. 1787-1794, 2011.

[12] S. Roperto, V. Russo, A. Özkul et al., "Bovine papillomavirus type 2 infects the urinary bladder of water buffalo (Bubalus bubalis) and plays a crucial role in bubaline urothelial carcinogenesis," Journal of General Virology, vol. 94, pp. 403-408, 2013.

[13] G. Borzacchiello, V. Russo, F. Gentile et al., "Bovine papillomavirus E5 oncoprotein binds to the activated form of the platelet-derived growth factor $\beta$ receptor in naturally occurring bovine urinary bladder tumours," Oncogene, vol. 25, no. 8, pp. 1251-1260, 2006.

[14] S. Roperto, R. de Tullio, C. Raso et al., "Calpain3 is expressed in a proteolitically active form in papillomavirus-associated urothelial tumors of the urinary bladder in cattle," Plos One, vol. 5, no. 4, Article ID e10299, 2010.

[15] J. N. Eble, G. Sauter, J. I. Epstein, and I. A. Sesterhenn, World Health Organization Classification of Tumours: Pathology and Genetics of Tumours of the Urinary System and Male Genital Organs, IARC Press, Lyon, France, 2004.

[16] A. M. Pamukcu, "Tumours of the urinary bladder," Bulletin of the World Health Organization, vol. 50, no. 1-2, pp. 43-52, 1974.

[17] R. Somvanshi, S. Pathania, N. Nagarajan, K. Pangty, and P. Kumar, "Pathological study of non-neolastic urinary bladder lesions in cattle and buffaloes: a preliminary report," Tropical Animal Healthy Production, vol. 44, no. 4, pp. 855-861, 2012.

[18] F. Aloisi and R. Pujol-Borrell, "Lymphoid neogenesis in chronic inflammatory diseases," Nature Reviews Immunology, vol. 6, no. 3, pp. 205-217, 2006.

[19] Y. Modis, B. L. Trus, and S. C. Harrison, "Atomic model of the papillomavirus capsid," EMBO Journal, vol. 21, no. 18, pp. 47544762, 2002.

[20] B. Marchetti, G. H. Ashrafi, E. Tsirimonaki, P. M. O’Brien, and M. S. Campo, "The bovine papillomavirus oncoprotein E5 retains MHC class I molecules in the Golgi apparatus and prevents their transport to the cell surface," Oncogene, vol. 21, no. 51, pp. 7808-7816, 2002.

[21] A. Viola, A. Sarukhan, V. Bronte, and B. Molon, "The pros and cons of chemokines in tumor Immunology," Trends in Immunology, vol. 33, pp. 496-504, 2012.

[22] R. M. Peek, S. Mohla, and R. N. DuBois, "Inflammation in the genesis and perpetuation of cancer: summary and recommendations from a National Cancer Institute-sponsored meeting," Cancer Research, vol. 65, no. 19, pp. 8583-8586, 2005. 


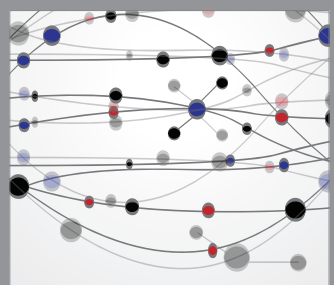

The Scientific World Journal
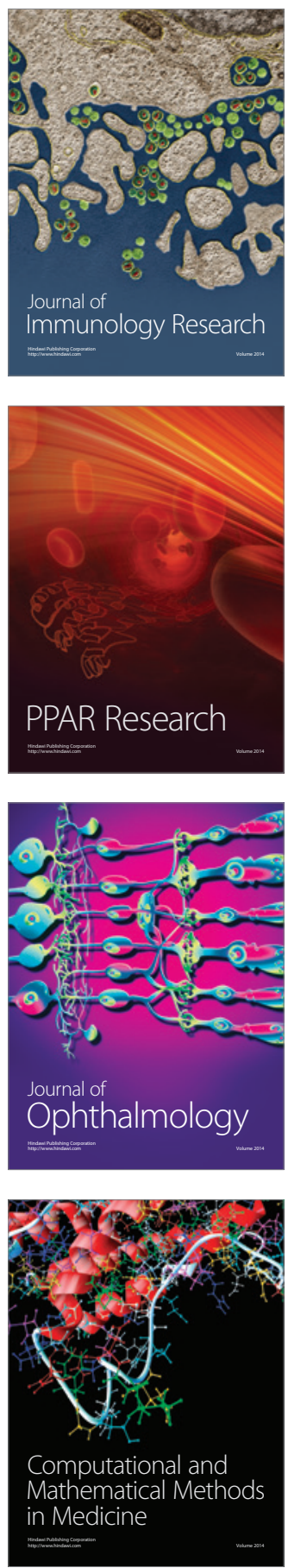

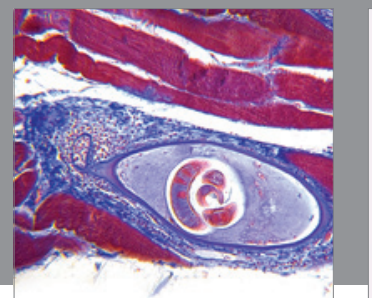

Gastroenterology

Research and Practice
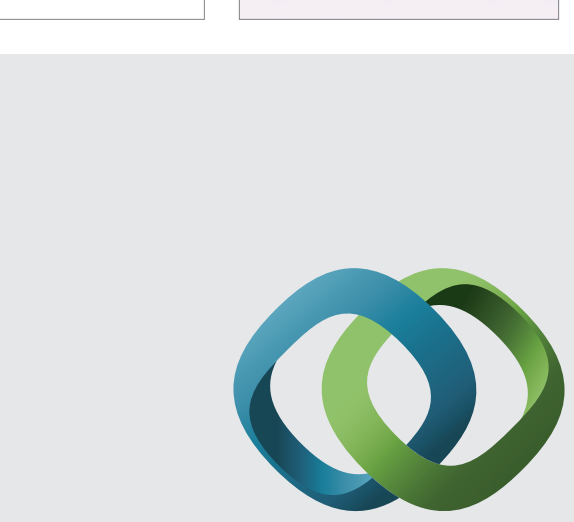

\section{Hindawi}

Submit your manuscripts at

http://www.hindawi.com
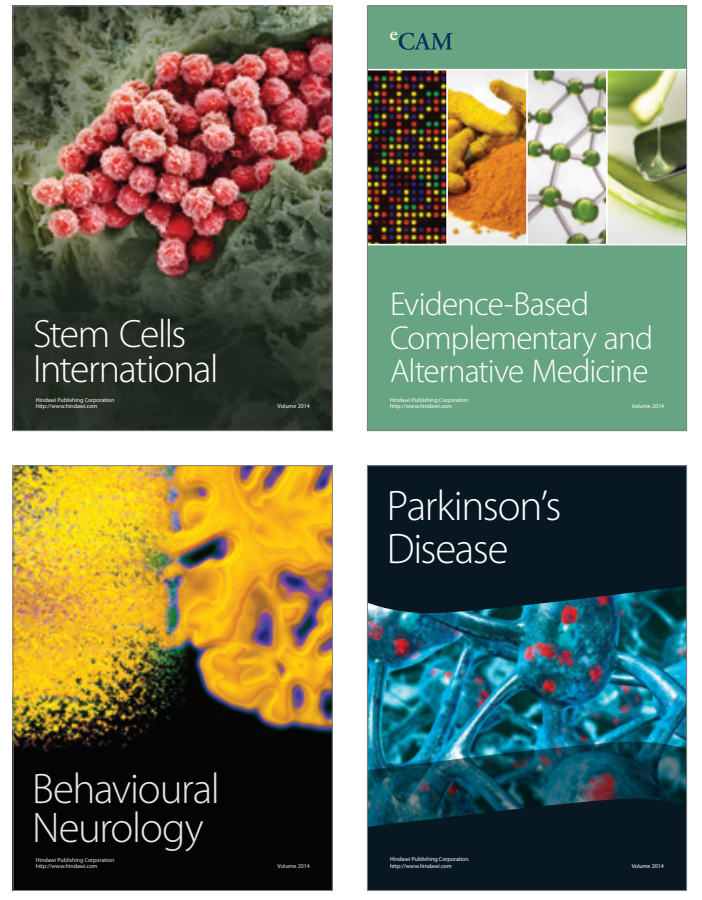
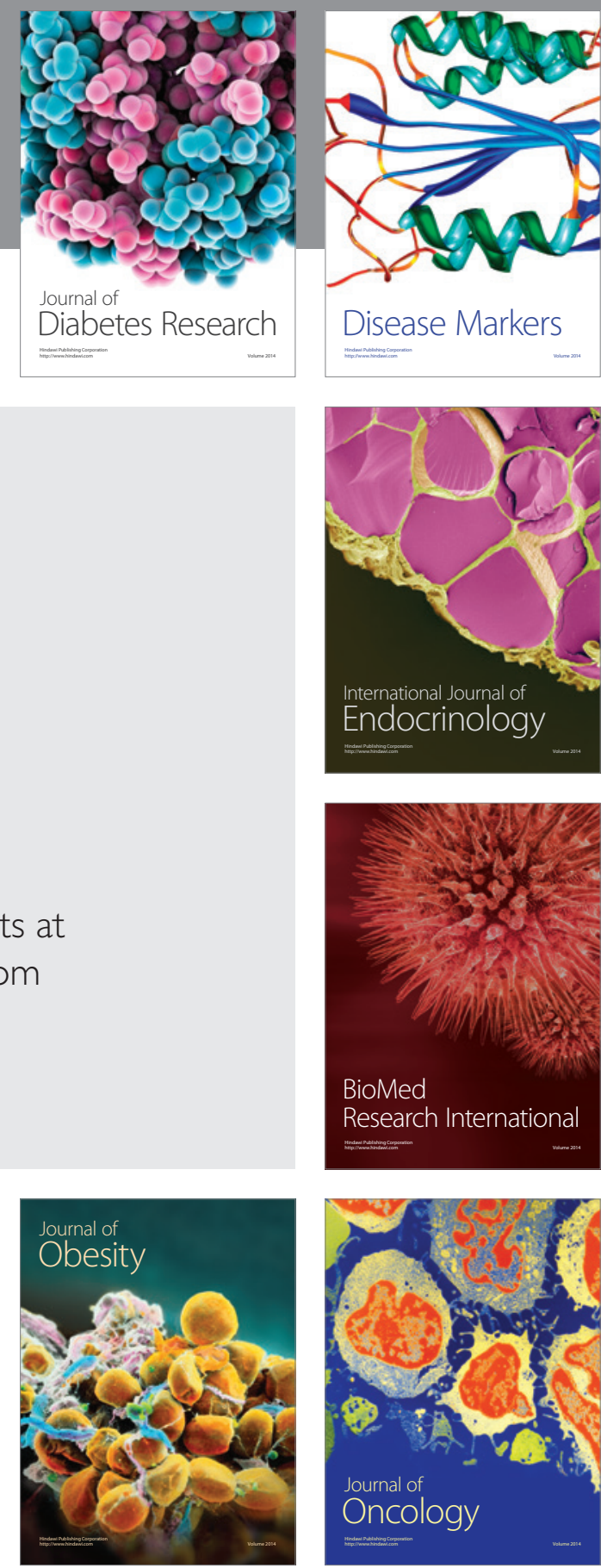

Disease Markers
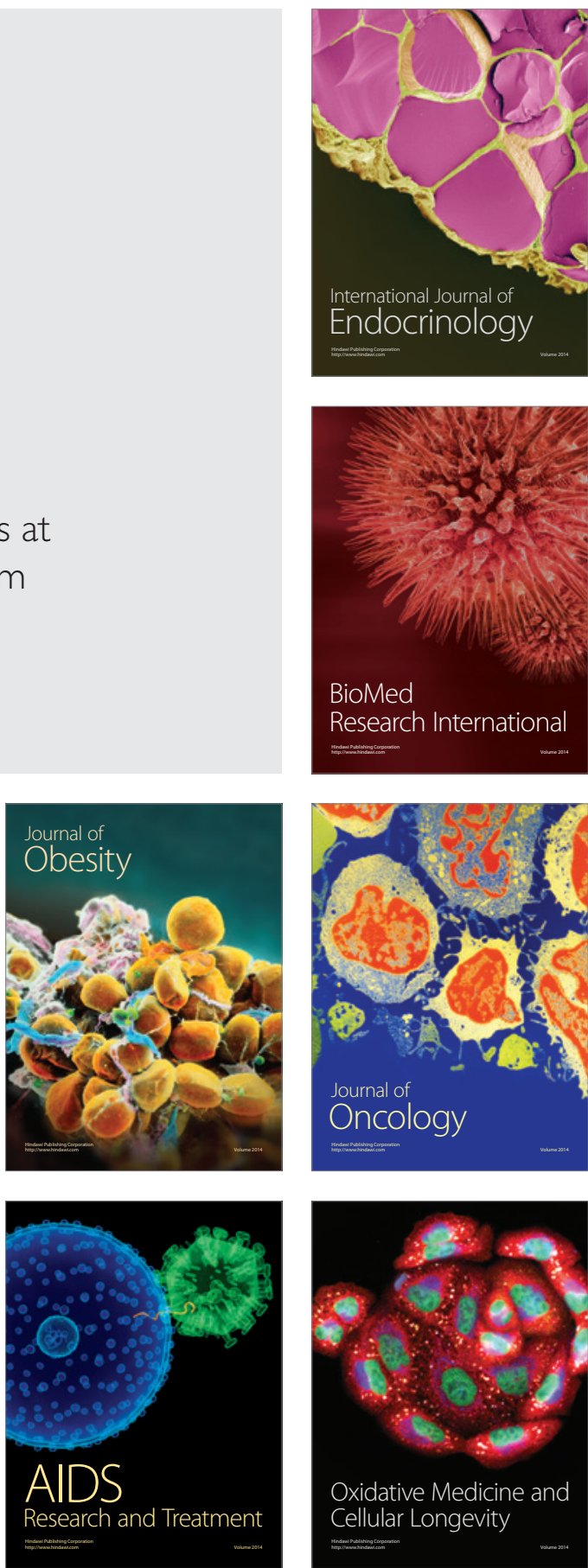